\title{
Here Today, Gone Tomorrow? Changes in 4-Month-Olds' Physiologic and Behavioral Responses Do Not Indicate Memory for a Social Stressor
}

\author{
Jennifer A. DiCorcia*, Nancy C. Snidman and Ed Tronick \\ Department of Psychology, University of Massachusetts, Boston, MA, United States
}

OPEN ACCESS

Edited by:

Caspar Addyman,

Goldsmiths, University of London,

United Kingdom

Reviewed by:

Eric A. Walle,

University of California, Merced

United States

Sam Wass,

University of Cambridge,

United Kingdom

*Correspondence:

Jennifer A. DiCorcia

jennifer.dicorcia@umb.edu

Specialty section: This article was submitted to

Developmental Psychology,

a section of the journal

Frontiers in Psychology

Received: 28 September 2017

Accepted: 26 January 2018

Published: 20 February 2018

Citation:

DiCorcia JA, Snidman NC and Tronick E (2018) Here Today, Gone

Tomorrow? Changes in

4-Month-Olds' Physiologic and Behavioral Responses Do Not Indicate

Memory for a Social Stressor.

Front. Psychol. 9:128.

doi: 10.3389/fpsyg.2018.00128
Although much is known about early memory development, only a few studies have explored infants' memory of social stress. While these few studies suggest that infants can remember stressful interactions, limitations seen in both methodology and statistical analyses give pause. In the current study, 4-month-olds and their mothers participated in both stressful and non-stressful interactions over 2 days. On Day 1, memory group infants participated in the double Face-to-Face Still-Face (FFSF) paradigm and control group infants participated in typical play. Both groups experienced the double FFSF paradigm on Day 2. Memory group infants exhibited the standard SF response but no differences in infant cortisol on Day 1. Both infant groups exhibited the standard SF response on Day 2. However, infants in the memory group, who saw the FFSF paradigm for the second time, did not demonstrate changes in cortisol or behavior indicative of memory across the 2 days. There was also no relationship between changes in cortisol and behavior for both days. The findings question the use of salivary cortisol as a measure of social stress and suggest that, although 4-month-olds reacted to the Still-Face social stressor immediately, they did not remember the following day.

Keywords: infant memory, social stress, salivary cortisol, infant behavior, double Face-to-Face Still-Face (FFSF) paradigm

\section{INTRODUCTION}

The age at which infants and young children remember events is a topic of considerable interest to developmental and cognitive psychology, as well as to brain development and psychobiology. A large and important literature has found an increase in memory capacity with age and repeated exposure (Rovee-Collier, 1999; Carver and Bauer, 2001; Giles and Rovee-Collier, 2011; Lukowski and Bauer, 2013). However, the majority of this research is limited to infants' memory of people, objects, and actions. For example, in the first half year of life infants' recognize familiar faces (Pascalis and de Schonen, 1994) and their memory for specific actions increases from a few days to a few weeks (Hartshorn et al., 1998; Rovee-Collier, 1999). Infants also show the beginnings of deferred imitation (Pascalis and de Schonen, 1994; Learmonth et al., 2004). Considerably less is known about infants' developing memory for socioemotional experiences, specifically social stress. With the growth of 
the social neurosciences and the recognition of the fundamental importance of social experience for development, a question emerges as to whether memory for social stress, is similar to, partially overlapping, or even distinctly different than, memory for objects, events, and actions. Surprisingly only a few studies have looked at infants' memory of social stressors, and their findings, while intriguing, do not form a consistent picture due to different ages, methods, and indices of memory (Haley et al., 2011; Montirosso et al., 2013). Building on our own work and the work of others, in this study 4-month-old infants' memory for the maternal Still-Face (SF), an established infant social stressor, is examined using both behavioral and adrenocortical measures of memory.

The Face-to-Face Still-Face (FFSF) paradigm has consistently been a particularly fruitful methodological tool for evaluating young infants' reactivity and regulatory capacities and their ability to cope with an interactive stressor, the maternal Still-Face (SF) (Tronick et al., 1978; Cole et al., 2004). The original FFSF paradigm consisted of three 2-min episodes, face-to-face play, the SF, and another face-to-face play often referred to as the reunion episode (Tronick et al., 1978). Behaviorally, infants typically respond to the SF with what has come to be called the signature "still-face effect" (Adamson and Frick, 2003), a signature decrease in positive affect and an increase in negative affect and gaze aversion (Stack and Muir, 1990; Toda and Fogel, 1993; Weinberg and Tronick, 1996). In studies that have used micro-analytic scoring systems, infants have shown additional signs of distress in response to the SF with an increase in visual scanning, pickme-up gestures, distancing behavior such as twisting and turning in their seat, and autonomic stress indicators such as spitting up (Toda and Fogel, 1993; Weinberg and Tronick, 1996; Weinberg et al., 1999). A number of physiologic and neuroendocrine measures have also been used to measure infants' reactions during the FFSF paradigm. Ramsay and Lewis (2003) measured infant salivary cortisol and found that 6-month-old infants had a significant increase in salivary cortisol in response to the SF, though only about half of the infants showed a cortisol increase. In an attempt to increase infant stress, Haley and Stansbury (2003) modified the standard FFSF paradigm to include two SF episodes (i.e., play, SF, play, SF, play). This modified procedure did elicit a significant change in cortisol, albeit modest, following the SF stressors.

While there are an abundance of studies that have examined the FFSF paradigm's immediate effect on infant behavior and physiologic systems, to the authors' knowledge only two studies have used similar indices of memory to examine the paradigm's lasting effects (Haley et al., 2011; Montirosso et al., 2013). These two studies differ from one another in critical ways that add to our current understanding of infants' memory, but each is not without its own weakness. Haley et al. (2011) found an anticipatory stress response in 6-month-old's response to the double FFSF paradigm following a 24-h delay. In their study during the first lab visit infants were separated into two groups, one group that participated in the double FFSF paradigm and another group that engaged in typical play with their mothers. The inclusion of the control group is an important feature of the study as it controls for environmental stress from the testing experience. Both groups returned to the lab the next day to see if the FFSF group anticipated the same stressful experience. Although there were no behavioral differences indicative of memory, as expected, infants exposed to the SF had greater changes in salivary cortisol on the second day compared to the control group. While the comparison between the memory group and control group is an important comparison, the authors failed to discuss changes in cortisol across days. That is, were the infants who showed an increase in cortisol on the second day the same group of infants who also showed an increase on the first day? Overlooking the pattern of cortisol reactivity between days dismisses the importance of individual differences in reactivity. Recent reviews suggest great variability in infants' cortisol reactivity highlighting the importance of exploring individual differences in reactivity instead of relying on mean differences (Gunnar et al., 2009; Jansen et al., 2010; Tollenaar et al., 2010). Additionally, having all infants partake in the double FFSF paradigm on the second day (i.e., second exposure to the paradigm for the memory group and first exposure for the control group), would have strengthened the interpretation of the comparison between groups on the second day. Nonetheless, despite the limitations, the study is important for its demonstration of changes in salivary cortisol as a potential anticipatory measure of infant memory.

The methodological limitations seen in Haley et al. (2011) were partially addressed in another study that also explored 4-month-old infants' memory for the double FFSF paradigm. Montirosso et al. (2013) observed changes in infant cortisol and behavior in response to the double FFSF paradigm following a 2 week delay. Unlike the Haley study, infants' reactivity to the FFSF paradigm was again measured during the second visit. The repeated exposure to the FFSF paradigm for the memory group along with the inclusion of a control group that experienced the paradigm once allowed for both within and between participant comparisons. However, the control group only visited the lab once and any changes in infants' behavior and cortisol reactivity may have been due to the FFSF paradigm itself, to the novel experience of visiting the lab, or both. Like Haley and colleagues, Montirosso and colleagues found no differences in behavior between the two visits, but they did not find significant differences in cortisol reactivity between groups perhaps due to the longer memory delay ( $24 \mathrm{~h}$ compared to 2 weeks) or age differences (6-month-olds compared to 4-month-olds). As a next step, individual differences in cortisol reactivity were considered by dividing memory group infants into two cortisol reactivity groups, increasers and decreasers, based upon infants' greatest change in cortisol following their first exposure to the paradigm. Once again behavioral differences were not found between cortisol reactivity groups, but there were differences in cortisol reactivity during infants' second exposure to the paradigm. During their second exposure to the paradigm, increasers again showed an increase in cortisol but to a lesser extent while decreasers now had increased cortisol. These differences across exposures were interpreted as memory. However, this study too was not without limitations. First, the control group visited the lab once. Perhaps the change in cortisol for the subsample of the memory group was due in part to the novelty of the lab 
experience and not in response to the social stressor. Variability in cortisol reactivity might be because of individual differences within the infant. In fact, several recent reviews question the use of cortisol as a measure of social stress in infants since most studies lack overall significant changes in cortisol and instead rely on the creation of reactivity groups (Gunnar et al., 2009; Jansen et al., 2010). This is an important point to consider because any difference between post-cortisol and baseline-no matter how small-was used to create cortisol reactivity groups in the Montirosso study. Day-to-day variation and assay error were not accounted for which may have overinflated the stress responder group. The observed changes need to exceed error of measurement, at the very least, to create reactivity groups. Methodologic recommendations for salivary cortisol suggest that differences need to be greater than $\pm 0.02 \mu \mathrm{g} / \mathrm{dL}$ and postparadigm cortisol must be at least $10 \%$ different than baseline in order to differentiate between an actual significant change in response to the stressor and assay error (Granger et al., 2012).

Even with their limitations, the findings from these initial studies suggest a developing capacity to remember social stress. Building upon past research, in the current study infants and their mothers were randomly assigned to either an experimental memory group (Group Memory) or a control group (Group Control $_{\text {) }}$. Dyads assigned to Group Memory $_{\text {experienced the double FFSF }}$ paradigm on Day 1 and again on Day 2. Group Control dyads experienced five 2-min episodes of face-to-face play with their mothers on Day 1 and the double FFSF paradigm on Day 2. Memory for the social stressor was assessed using changes in infant cortisol and behavior. The current study adds to previous research in several important ways. First, the current study employed a balanced research design where infants in both groups were tested twice over $24 \mathrm{~h}$. This design offered two key memory comparisons-a between group comparison within each day and a within group comparison across the 2 days.

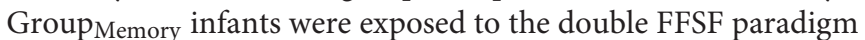
twice which allows for a test of memory capacity and stability of responses. Group Control infants were also tested twice and served as a control comparison in two important ways. Their Day 1 experience controlled for possible behavioral or physiological effects associated with visiting a research lab (e.g., visiting a new place, meeting new people, use of physiological sensors, etc.) and on Day 2 their first experience with the double FFSF paradigm served as a memory control. Finally, like Montirosso et al. (2013), individual differences in cortisol reactivity during infants' first visit to the lab were explored. However, in the current study error attributed to cortisol sampling was accounted for in the creation of Stress Response and No Stress Response groups.

Three main questions were asked of the data, (1) did infants show the established SF behavioral response and typical increase in cortisol during their first exposure to the double FFSF paradigm, (2) were there changes in behavior and cortisol indicative of memory for the paradigm following a $24 \mathrm{~h}$ delay, and (3) did individual differences in infants' cortisol reactivity during their first exposure to the paradigm relate to changes in behavior indicative of memory during their second exposure to the paradigm? Taken together, like previous studies, it was hypothesized that all infants would show behaviors and physiology indicative of stress in response to their first exposure to the double FFSF paradigm (decreased positive affect and increased negative affect and gaze aversion, increased cortisol) (Adamson and Frick, 2003) (Haley and Stansbury, 2003; Lewis and Ramsay, 2005). In response to their second exposure to the paradigm, GroupMemory infants were expected to show a change in physiology and behavior from their Day 1 initial reaction to the paradigm.

\section{METHOD}

\section{Participants}

Participating infant-mother dyads were recruited from the newborn nursery of a large Boston metropolitan area hospital that serves a diverse population. All infants were full-term, clinically normal at delivery and healthy at the time of their visit. Sixty-four infants (48\% female) and their mothers participated in the study (Group Memory $n=44$, Group Control $n=20$ ). Fourteen dyads were excluded from the study entirely due to maternal SF violations $(n=5)$, missing maternal video for SF violations assessment $(n=1)$, excessive infant crying during baseline play $(n=1)$, missing infant baseline and/or all post-paradigm cortisol samples $(n=5)$, and infants taking medications that affect cortisol levels $(n=2)$ (Hibel et al., 2006; Granger et al., 2009) which resulted in a final sample of fifty (Group Memory $n=33$, Group $\left._{\text {Control }} n=17\right)$ 4-month-old infants ( $M=111.86$ days, $S D=2.515$ ) and their mothers (see Table 1 for demographics). Families were provided with a small stipend and infant gift for their participation. The study was approved by the University's IRB and all mothers provided written informed consent.

\section{Procedure}

Mothers and their 4-month-old infants visited the laboratory (Child Development Unit) twice over 2 consecutive days. To

TABLE 1 | Maternal demographics.

\begin{tabular}{|c|c|c|c|c|}
\hline & \multicolumn{2}{|c|}{ Group Memory } & \multicolumn{2}{|c|}{ Group Control } \\
\hline & $n$ & $\%$ & $n$ & $\%$ \\
\hline \multicolumn{5}{|l|}{ ETHNICITY } \\
\hline Hispanic/Latino & 2 & 6 & 1 & 6 \\
\hline Non-hispanic & 31 & 94 & 16 & 94 \\
\hline \multicolumn{5}{|l|}{ RACE } \\
\hline Asian & $3^{\#}$ & 9 & 0 & 0 \\
\hline Black or African American & 2 & 6 & $3^{\#}$ & 18 \\
\hline White & 33 & 85 & 13 & 76 \\
\hline Not Reported/Declined & 0 & 0 & 1 & 6 \\
\hline \multicolumn{5}{|c|}{ HIGHEST EDUCATION OBTAINED } \\
\hline High school & 1 & 3 & 1 & 6 \\
\hline Some college & 1 & 3 & 2 & 12 \\
\hline College degree & 12 & 36 & 5 & 29 \\
\hline Graduate school & 19 & 58 & 9 & 53 \\
\hline
\end{tabular}

\#Two mothers in Group Memory identified as Asian and White. One mother in Group Control identified as Black or African American and White. 
keep time of day consistent for cortisol samples both within and across participants, infants were tested at the same time of day $( \pm 1 \mathrm{~h})$ during both visits and, regardless of group, all infants were tested in the morning/early afternoon hours (between 8:30 a.m. and 1 p.m.). Two research assistants conducted each testing session, a primary research assistant who interacted with the mother and infant and a secondary research assistant who was responsible for the behind-the-scenes technical equipment (e.g., cameras, timing the paradigm). The primary research assistant was the same person for both testing days. Dyads were randomly assigned to either an experimental memory FFSF group (Group Memory) or a control group (Group Control $_{\text {) on }}$ Day 1. Upon arrival, mothers and their infants were escorted to a waiting room where a research assistant explained the study and answered any questions. Before obtaining written consent, mothers were also reminded that they may terminate the procedure at any time for any reason. Approximately 20-30 min after arriving at the lab and following consenting procedures, two baseline saliva samples were collected from the infant, each $\sim 8 \mathrm{~min}$ apart. Dyads were then escorted to the testing room by a research assistant. Infants were placed in a highchair that was situated $\sim 46 \mathrm{~cm}$ directly in front of their mothers. To allow unrestricted maternal touch during play episodes the highchair did not have a tray. Following techniques developed for the FFSF procedure, two cameras were positioned (one mother, one infant) to record the testing session (Weinberg et al., 1999). Video recordings were synchronized using an integrated system and software packages [MindWare ACQ software (MindWare Technologies, Ltd., Westerville, $\mathrm{OH}$ ) and Mangold VideoSyncPro (Mangold International GmbH (Ed.)]. Before leaving the testing room the research assistant fit mothers with a small earpiece connected to a walkie-talkie so that she could discretely communicate with mothers during the testing session.

All dyads, regardless of group or day, participated in five, 2-min face-to-face episodes. To start the testing session, the research assistant instructed mothers to briefly look at a sign located on the wall to their right and then turn back to their infant. The sign served as a reminder to mothers about the study procedure and served as an episode starting cue for later video coding. Mothers then moved a yellow smock that hung on their back frontwards to their chest (in view of their infant) signaling the start of the testing session. The yellow smock served as a memory cue for Day 2. All mothers then started with an initial baseline play episode (Ep1 $\left.1_{\text {Play }}\right)$ in which the research assistant instructed them to play in an unstructured way with their infants as they typically would at home. While mothers were allowed to touch and talk to their infants during play sessions, they were not allowed to introduce any outside toys or props and were asked to refrain from picking their infant up during the testing session. Infants who cried continuously for more than $30 \mathrm{~s}$ during the first baseline play were excluded from the study. All five episodes ended with a short break (2-3s) during which mothers were instructed to stop what they were doing and look toward a sign located on their right. RAs then provided instructions for mothers to begin the next episode. This break ensured that both groups experienced five distinct episodes. This general procedure was repeated for both Group Memory and Group Control dyads for a total of the five 2-min episodes on both Day 1 and Day 2.

\section{GroupMemory}

Dyads randomly assigned to GroupMemory participated in the double FFSF paradigm both on Day 1 (first exposure) and Day 2 (second exposure). For both days, following Ep1 1 play, mothers were instructed to begin the first 2-min SF episode $\left(\mathrm{Ep} 22_{\mathrm{SF} 1}\right)$. Mothers were asked to sit back in their chair and to maintain a neutral expression or "poker-face" while looking at their infant. Mothers were asked to refrain from talking to and/or touching their infant. To ensure fidelity, video recordings of mothers were reviewed for violation of the SF instructions. Mothers who violated the SF procedure were excluded from the study (e.g., changed from a neutral expression, touched, or otherwise interacted with their infant for more than a total of $20 \mathrm{~s}$ ). Ep2 ${ }_{S F 1}$ was followed by another 2-min face-to-face play episode $\left(\mathrm{Ep} 3_{\text {Play }}\right)$, a second SF episode $\left(\mathrm{Ep} 4_{\mathrm{SF} 2}\right)$, and a final play episode (Ep5 $\left.5_{\text {Play }}\right)$.

\section{Group Control}

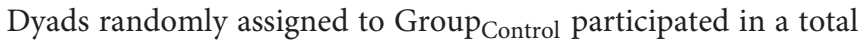
of five, 2-min face-to-face play episodes (Ep1 play to Ep5play) during their Day 1 visit. Dyads participated in the double FFSF paradigm during their Day 2 visit.

Group Control served as a control in two different ways. First as a control for possible behavioral or physiological effects associated with visiting a research lab (e.g., visiting a new place, meeting new people, use of physiological sensors, etc.) but without experiencing the additional stress of the SF. Second, as a memory control in order to compare dyads experiencing the double FFSF paradigm either on just 1 day or on both days. Compared to past research that lacked a similar control group (Haley et al., 2011; Montirosso et al., 2013) the addition of the all play control group allowed for several unique comparisons. On Day 1 GroupMemory dyads experienced the stress of the SF and

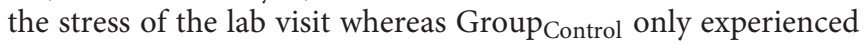
the stress of the lab visit. Thus, the comparison of Group Memory infants' reactivity on Day 1 to Group Control infants' reactivity speaks to the effect of the SF in addition to the stress of the visit. On Day 2 both groups experienced the stress of both the SF and the lab visit, but GroupMemory infants experienced the SF for the

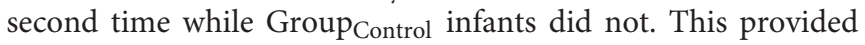
two ways of exploring memory for the SF, first by comparing Day 2 performance across groups and by comparing the performance of Group Memory infants across both days.

\section{Coding}

\section{Behavioral Coding}

Infant and mother behavior was videotaped and later coded by coders who were blind to study hypotheses and group membership. Gaze Faze, a modified Infant and Caregiver Engagement Phases (ICEP) coding system (Weinberg and Tronick, 1999), was used in which affect and gaze behaviors were coded separately. Gaze (toward mother for infant, toward infant for mother) along with positive (e.g., smiling, raised cheeks, 
and crinkly-eyes), negative (e.g., fussing, crying, frowning), and neutral affect were continuously coded using 1-s time intervals for both mother and infant using Mangold Interact software [Mangold International, GmbH (Ed)]. Mothers' and infants' faces must be visible for the majority of the episode time. Coders overlapped on at least $25 \%$ of total video files to evaluate interrater reliability (infant affect percent agreement $=75 \%$, Cohen's $\kappa=0.58$, infant gaze percent agreement $=73 \%, \kappa=0.62$ infant gaze).

\section{Cortisol}

Using a pre/post-stress design, four saliva samples were collected from infants. Saliva samples were collected with Sorbettes (Salimetrics, State College, PA), specifically designed for infants and small children. Two baseline samples were taken $8 \mathrm{~min}$ apart before the start of the testing paradigm followed by two post-paradigm samples taken at 20 and $35 \mathrm{~min}$ following the middle of the paradigm (i.e., the middle of Ep3 3 Play). Twenty and $35 \mathrm{~min}$ were chosen to reflect reactivity and recovery of cortisol, respectively (Ramsay and Lewis, 2003; Gunnar et al., 2009).

Saliva samples were immediately stored in a $-20^{\circ} \mathrm{C}$ degree freezer before being moved to $\mathrm{a}-80^{\circ} \mathrm{C}$ degree freezer for longerterm storage until shipped overnight on dry-ice for assay at the Institute for Interdisciplinary Salivary Bioscience. On the day of assay samples were thawed and centrifuged (to remove mucins) and assayed in duplicate for salivary cortisol using a commercially available immunoassay specifically designed for use with saliva without modification to the manufacturer's (Salimetrics, Carlsbad, CA) recommended protocol. The test volume was $25 \mu \mathrm{L}$, the range of sensitivity was from 0.007 to $3.0 \mu \mathrm{g} / \mathrm{dL}$, and intra- and inter-assay coefficients of variation were on average less than 10 and $15 \%$ respectively.

\section{Data Preparation}

Cortisol samples were excluded for many methodologic reasons including missing samples (due to extreme infant distress), insufficient sample volume, and assay interference. Samples were also excluded if infants were fed within $10 \mathrm{~min}$ of the sample time. While rinsing infants' mouths with water should reduce any potential effects of milk or formula, doing so in close proximity to the sample time may dilute cortisol concentrations, as per assay manufacturer's guidelines (Salimetrics, LLC). After individual cortisol samples were excluded, participants needed at least one remaining baseline and one of the two postparadigm cortisol samples (either 20 or $35 \mathrm{~min}$ ) to be included in the study. As a result, a final sample of 33 GroupMemory infants and 17 Group $_{\text {Control infants were included in cortisol }}$ only analyses. In addition to individual cortisol samples, two dependent variables were used to measure infant cortisol. In the first approach, since the specific time course for cortisol reactivity and recovery continues to be contested, infants' average baseline cortisol and highest peak cortisol were used to calculate a peak cortisol reactivity difference (i.e., average baselinegreatest peak post-paradigm sample). The second approach generated a cortisol reactivity category for each infant where baseline and peak post-stress cortisol values were used to categorize infants. The difference between the greatest postparadigm cortisol sample and the average baseline sample needed to be both positive and greater than $10 \%$ for an infant to be classified as having a Stress Response and being different enough from the intra-assay coefficient of variation for the sample (Granger et al., 2012). If the difference was negative and/or less than $10 \%$, the infant was classified as No Stress Response. This categorization approach differed from those used in previous work that categorized any increase, regardless of its size, as a responder without accounting for potential sample error attributed to the assay (Montirosso et al., 2013).

For behavioral data, duration of time was changed to proportion of time to account for differences in total episode times (e.g., episodes that were shortened due to extreme infant distress). Testing sessions were stopped early for infants who continuously cried for more than $30 \mathrm{~s}$ at any time after Ep $1_{\text {Play }}$ (Group $_{\text {Memory }} n=5$, Group Control $n=2$ ). There was no relationship between study drop-outs and group membership (Group $_{\text {Memory }}=15 \%$, Group $\left._{\text {Control }}=12 \%, p>0.99\right)$. As a more conservative approach to the data, these seven dyads were dropped from behavioral analyses due to missing episode data. As a result, a final sample of 28 Group Memory infants and 15 Group Control infants and were included in behavioral analyses.

All data were explored within groups for possible univariate ( $> \pm 3 S D$ from the mean) and multivariate (Mahalanobis Distance, $p<0.001$ ) outliers in addition to violations of normality across all dependent variables. To maintain as many data points as possible, outliers were kept and winsorized to $\pm 3 S D$ s from the mean. There were no differences in overall findings for analyses conducted with winsorized values or without outliers. Behavioral data was transformed using an arcsine transformation appropriate for proportion data $\left(2^{*}\left(\operatorname{arcsine}\left(\mathrm{p}^{1 / 2}\right)\right)\right.$ to correct for normality issues (Howell, 2002). Cortisol values were corrected for normality using the common $\log \left(\log _{10}\right)$. Transformed data were used in analyses while raw data were used for descriptive statistics presented in figures and tables for clarity.

\section{DESIGN AND ANALYSIS}

Preliminary results revealed no significant gender main effects or interactions so gender was not considered as a factor. Infant cortisol and behavioral data were analyzed using a series of univariate and mixed factorial GLMs with Group (Group Memory, Group Control), Day (Day 1, Day 2), Sample

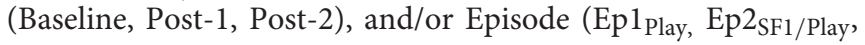
Ep3 $3_{\text {Play }}$, Ep4 $4_{\text {SF2/Play }}$, Ep5 $5_{\text {Play }}$ ) as factors. Multivariate results were used to address sphericity issues. A priori comparisons were explored using Bonferroni corrections [adjusted alpha levels $=0.0125$ (two-way interaction), 0.001 (three-way interaction)]. Changes in infant cortisol were also explored using $t$-Tests and cortisol categories were explored using non-parametric analyses. Several questions were asked of the data related to Group Memory infants' first exposure to the double FFSF paradigm and their memory of the paradigm following a $24 \mathrm{~h}$ delay. 


\section{RESULTS}

\section{Question 1. Did Group Memory Infants Show the Established SF Response and Typical Increase in Cortisol in Response to Their First Exposure to the Paradigm? Infant Behavior}

As an initial step, Group Memory infants' first encounter with the double FFSF paradigm was analyzed before exploring their memory for the social stressor. A significant Episode main effect was found for each behavioral variable (see Table 2). As shown in Table 3, infants' exhibited the SF effect. Negative affect increased in response to the SF episodes and then subsequently decreased during play episodes while positive affect and infants' gaze to their mothers showed the opposite pattern. Although not typically reported as part of the SF effect, infants' neutral affect generally decreased over time. Infants were also more negative during the second SF compared to the first SF (Ep2 $2_{\mathrm{SF} 1}$ vs. Ep4 $4_{\mathrm{SF} 2}: M_{D}=$ $\left.-0.528, S E_{D}=0.088, p<0.001\right)$.

\section{Infant Cortisol}

Three analytic approaches were used to explore changes in cortisol following Group Memory infants' initial response to the double FFSF paradigm on Day 1. First, there was no difference between Group Memory infants' baseline cortisol and their greater post-paradigm cortisol, $\mathrm{F}_{(1,32)}=0.007, p=0.933, \eta_{p}{ }^{2}<0.001$ (see Table 4 for descriptive statistics). As a second approach,

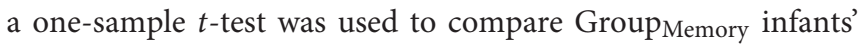

TABLE 2 | Statistical findings for Group Memory infants' behavior during their first exposure to the double FFSF paradigm.

\begin{tabular}{lcccc}
\hline \multicolumn{5}{c}{ Episode } \\
\hline Dependent variable & Wilks ' $\lambda$ & $\boldsymbol{F}_{(\mathrm{df})}$ & $\boldsymbol{p}$ & $\eta_{\boldsymbol{p}}{ }^{2}$ \\
\hline Gaze to mother & 0.123 & $46.230(4,26)$ & $p<0.001$ & $\eta_{p}{ }^{2}=0.877$ \\
Negative affect & 0.308 & $14.611(4,26)$ & $p<0.001$ & $\eta_{p}{ }^{2}=0.692$ \\
Positive affect & 0.246 & $19.945(4,26)$ & $p<0.001$ & $\eta_{p}{ }^{2}=0.754$ \\
Neutral affect & 0.378 & $10.681(4,26)$ & $p<0.001$ & $\eta_{p}{ }^{2}=0.622$ \\
\hline
\end{tabular}

Group Memory infants demonstrated the established behavioral SF effect during their first encounter with the paradigm. peak cortisol reactivity difference to a $10 \%$ notable difference. Results once again suggested that infants' did not show a cortisol stress response, $t_{(32)}=-1.837, p=0.076, d=0.320$. A final approach explored whether Group Memory infants were more likely to be categorized as having a Stress Response than not. Equal numbers of GroupMemory infants were categorized as having a Stress Response (52\%) and as No Stress Response (48\%), $X_{(1, N=33)}^{2}$ $=0.030, p=0.862$.

\section{Question 2. Were There Changes in Behavior and/or Cortisol Indicative of Memory Following the $24 \mathrm{~h}$ delay? Was There Behavioral Evidence of Memory for the FFSF Paradigm?}

Two different comparisons were used to explore memory for the FFSF paradigm, (1) Group Control infants' behavior compared to Group Memory infants' behavior on Day 2, and (2) changes in Group ${ }_{\text {Memory infants' behavior from Day } 1 \text { to Day } 2 .}$

As a first approach, Group Memory infants' behavior on Day 2 (i.e., their second exposure to the FFSF paradigm) was compared to Group Control infants' behavior on Day 2 (i.e., their first exposure to the FFSF paradigm). Main effects and interactions are shown in Table 5. There were no differences in infant gaze $(p s>0.1)$ or infant positive affect $(p s>0.1)$ on Day 2 during any episode (see Figures 1, 2). Group Control infants and Group Memory infants looked at their mothers for the same amount of time and were equally positive on Day 2. For negative

\begin{tabular}{|c|c|c|c|c|c|c|}
\hline & \multicolumn{2}{|c|}{$\begin{array}{l}\text { Average } \\
\text { baseline }\end{array}$} & \multicolumn{2}{|c|}{$\begin{array}{c}\text { Greatest } \\
\text { peak }\end{array}$} & \multicolumn{2}{|c|}{$\begin{array}{c}\text { Peak } \\
\text { reactivity }\end{array}$} \\
\hline & Mean & $S D$ & Mean & $S D$ & Mean & $S D$ \\
\hline \multicolumn{7}{|l|}{ DAY 1} \\
\hline GroupMemory $_{(n=33)}$ & 0.394 & 0.320 & 0.427 & 0.418 & 0.033 & 0.271 \\
\hline $\operatorname{Group}_{\text {Control }}(n=17)$ & 0.257 & 0.191 & 0.305 & 0.262 & 0.048 & 0.305 \\
\hline \multicolumn{7}{|l|}{ DAY 2} \\
\hline GroupMemory $(n=33)$ & 0.444 & 0.413 & 0.493 & 0.508 & 0.050 & 0.334 \\
\hline $\operatorname{Group}_{\text {Control }}(n=17)$ & 0.404 & 0.844 & 0.570 & 1.278 & 0.166 & 0.480 \\
\hline
\end{tabular}

Raw cortisol values $(\mu \mathrm{g} / \mathrm{dL})$ are presented for clarity.

TABLE 3 | Mean differences and standard errors for behavioral variables following significant episode main effects.

\begin{tabular}{|c|c|c|c|c|}
\hline & Gaze to mom & Negative affect & Positive affect & Neutral affect \\
\hline Ep1Play vs. Ep2sF1 & $0.453(0.049)^{\star \star \star}$ & $-0.144(0.031)^{\star \star \star}$ & $0.253(0.039)^{\star \star \star}$ & $-0.108(0.051)^{\star}$ \\
\hline Ep2sF1 vs. Ep3Play & $-0.399(0.055)^{\star \star \star}$ & $0.066(0.031)^{\star \star}$ & $-0.212(0.040)^{\star \star \star}$ & $0.145(0.042)^{\star \star}$ \\
\hline Ep3Play vs. Ep4sF2 & $0.520(0.043)^{\star \star \star}$ & $-0.305(0.048)^{\star \star \star}$ & $0.225(0.043)^{\star \star \star}$ & $0.080(0.064)$ \\
\hline Ep4sF2 vs. Ep5play & $-0.415(0.059)^{\star \star \star}$ & $0.112(0.061)^{\#}$ & $-0.127(0.036)^{\star \star}$ & $0.032(0.051)$ \\
\hline
\end{tabular}

\# significant at $p<0.1$.

* significant at $p<0.05$.

**significant at $p<0.01$.

${ }^{* \star \star}$ significant at $p<0.001$. 


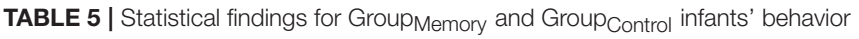
across episodes and days.

\begin{tabular}{lcccccccc}
\hline & \multicolumn{3}{c}{ Episode x Group } & & \multicolumn{3}{c}{ Episode x Group x Day } \\
\cline { 2 - 4 } & Wilks' $\lambda$ & $\boldsymbol{F}_{(1,41)}$ & $\eta_{\text {partial }}^{\mathbf{2}}$ & Wilks' $\lambda$ & $\boldsymbol{F}_{(4,38)}$ & $\eta_{\text {Partial }}^{2}$ \\
\hline Gaze to mother & 0.836 & $8.065^{\star \star}$ & 0.164 & 0.411 & $13.591^{\star \star \star}$ & 0.589 \\
Negative affect & 0.920 & $3.569^{\#}$ & 0.080 & 0.797 & $2.426^{\#}$ & 0.203 \\
Positive affect & 0.996 & 0.177 & 0.004 & 0.655 & $5.014^{\star \star}$ & 0.345 \\
Neutral affect & 0.855 & $6.979^{\star}$ & 0.145 & 0.907 & 0.975 & 0.093
\end{tabular}

\#significant at $<0.1$

*significant at 0.05 .

**significant at 0.01

${ }^{* \star *}$ significant at 0.001

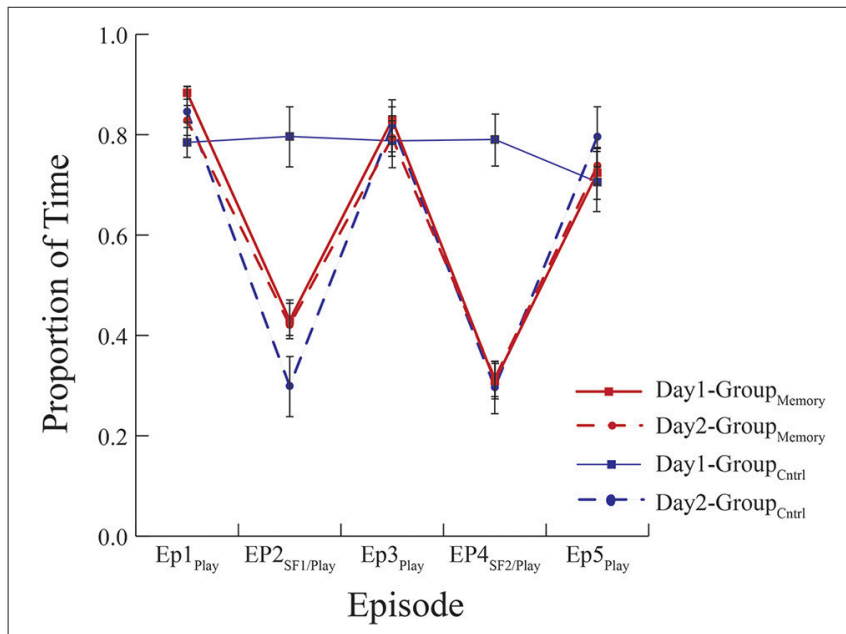

FIGURE 1 | Mean proportion of time infants spent looking at their mother. Group Memory infants spent less time looking at their mothers during the SF

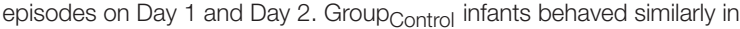
response to their first exposure to the FFSF on Day 2. There were no differences in behavior indicative of memory between infants' first and second exposures to the FFSF paradigm.

affect, Group ${ }_{\text {Control }}$ infants were more negative during Ep1 $1_{\text {Play }}$ $(p=0.002), \mathrm{Ep}_{\mathrm{SF} 1 / \text { Play }}(p=0.084)$, and Ep3 $3_{\text {Play }}(p=0.060)$ than Group Memory infants.

As a second approach, Group Memory infants' behavior was compared across episodes and days (see Table 6). There was a main effect of Episode for all variables. Regardless of day, infants once again demonstrated the SF effect. There was also a significant Day x Episode interaction for positive affect where infants were more positive during Ep $1_{\text {Play }}$ on Day 1 than Day $2\left(\mathrm{M}_{\mathrm{D}}=0.212, \mathrm{SE}_{\mathrm{D}}=0.075, p=0.009\right)$. Significant positive correlations between SF episodes across days were also found for most behavioral variables [Ep2 ${ }_{\mathrm{SF} 1}$ Day 1 compared to Day 2: Gaze $r_{(28)}=0.313, p=0.105$, Negative Affect: $r_{(28)}=0.350, p$ $=0.068$, Positive Affect: $r_{(28)}=0.449, p=0.017$, Neutral Affect: $r_{(28)}=0.282, p=0.146$; Ep4 $4_{S F 2}$ Day 1 compared to Day 2: Gaze $r_{(28)}=0.480, p=0.010$, Negative Affect: $r_{(28)}=0.338, p=0.079$, Positive Affect: $r_{(28)}=0.441, p=0.019$, Neutral Affect: $r_{(28)}=$ $0.321, p=0.096]$

\section{Was There Physiological Evidence of Memory for the FFSF Paradigm?}

Like infant behavior, two different comparisons were used to explore possible physiologic indices of memory for the FFSF paradigm, (1) Group Memory infants' Day 2 cortisol compared GroupControl infants' Day 2 cortisol and (2)

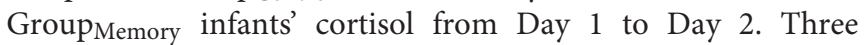
cortisol response variables were used, individual samples (average baseline, greater post-paradigm sample), peak cortisol reactivity difference (greatest post-paradigm peak-average baseline), and a cortisol reactivity category (Stress Response, No Stress Response). Descriptive statistics are shown in Table 4.

First, when comparing Group Memory $_{\text {to }}$ Group $_{\text {Control infants, }}$ the 2(Group) x 2(Day) x 2(Sample: Average BL, greater postparadigm sample) interaction was not significant, Wilks' $\lambda=$ 0.986, $F_{(1,48)}=0.670, p=0.417, \eta_{p}{ }^{2}=0.014$. Next, peak cortisol reactivity differences were compared across days and between groups. Once again, the 2(Group) x 2(Day) interaction was not significant, Wilks' $\lambda=0.984, F_{(1,48)}=0.793, p=$ $0.378, \eta_{p}^{2}=0.016$. As shown in Figure 3, although infants' peak cortisol reactivity difference increased from Day 1 to Day 2 for both groups, the increase was not significant. Finally, chisquare tests of independence were used to compare infants' stress response category within groups for Day 2. As shown in Figures $\mathbf{4 A , B}$, while there was no difference in stress response categories between groups for Day $1, X_{(1, N=50)}^{2}=0.089, p=$ $0.765, \Phi=0.042$, there was a marginally significant finding for Day 2, $X_{(1, N=50)}^{2}=3.566, p=0.059, \Phi=0.267$, where $71 \%$ of the Group $_{\text {Control infants were categorized as having a cortisol Stress }}$ Response compared to $42 \%$ of Group Memory infants.

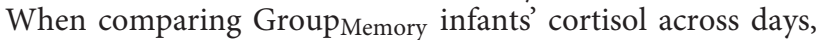
the 2(Day) x 2(Sample) interaction was not significant, Wilks' $\lambda=0.990, F_{(1,32)}=0.335, p=0.567, \eta_{p}{ }^{2}=0.010$. Peak cortisol reactivity differences were also compared across days and were not significantly different, $t_{(32)}=-0.579, p=0.567$, $\mathrm{d}=0.101$. Finally, a McNemar test compared GroupMemory infants' stress categorization across days and once again found no difference, $p=0.549$ (30\% Stress Response both days, 36\% No Stress Response both days, 21\% Stress Response Day 1 only, and $12 \%$ Stress Response Day 2 only). Similar to behavior, significant positive correlations were found between samples and days (see Table 7). There was also a significant positive correlation between Group Memory infants' peak cortisol reactivity difference across days, $r_{(33)}=0.395, p=0.023$.

\section{Question 3. Was There a Relationship between Infants' Cortisol Stress Reactivity Category on Day 1 and Their Behavior on Day 2 That Would Indicate Memory for the FFSF Paradigm?}

The final set of questions explored relationships between Group Memory infants' cortisol Stress Response Category on Day 1 (Stress Response, No Stress Response) and their behavior on Day 2. As shown in Table 8, there were no significant main effects 


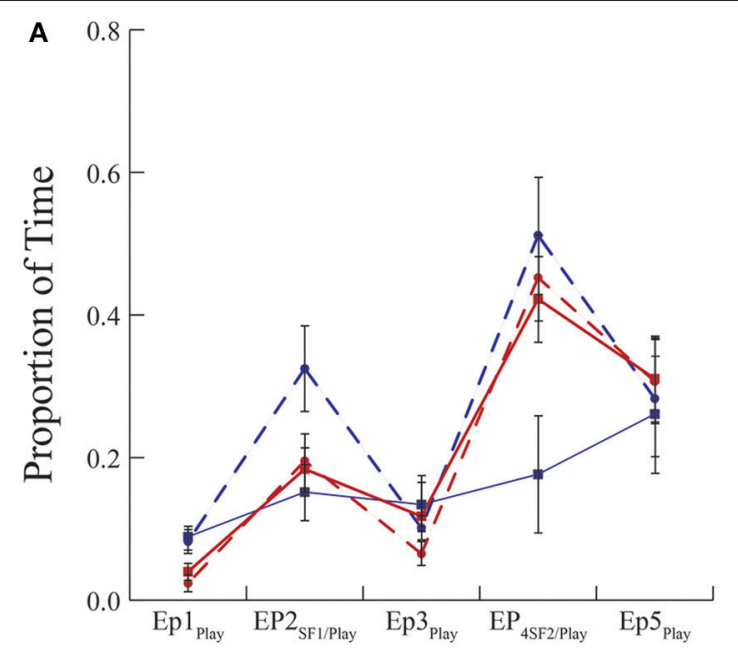

Episode

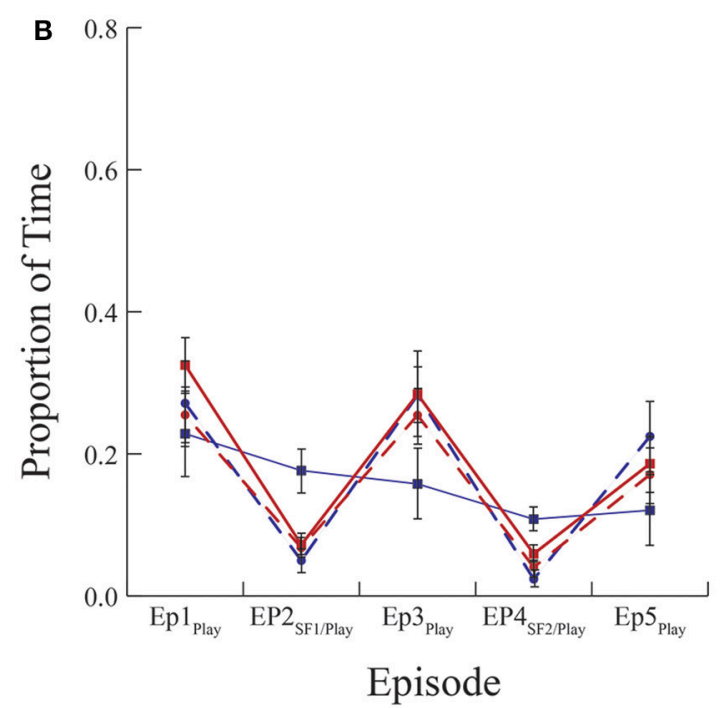

Episode

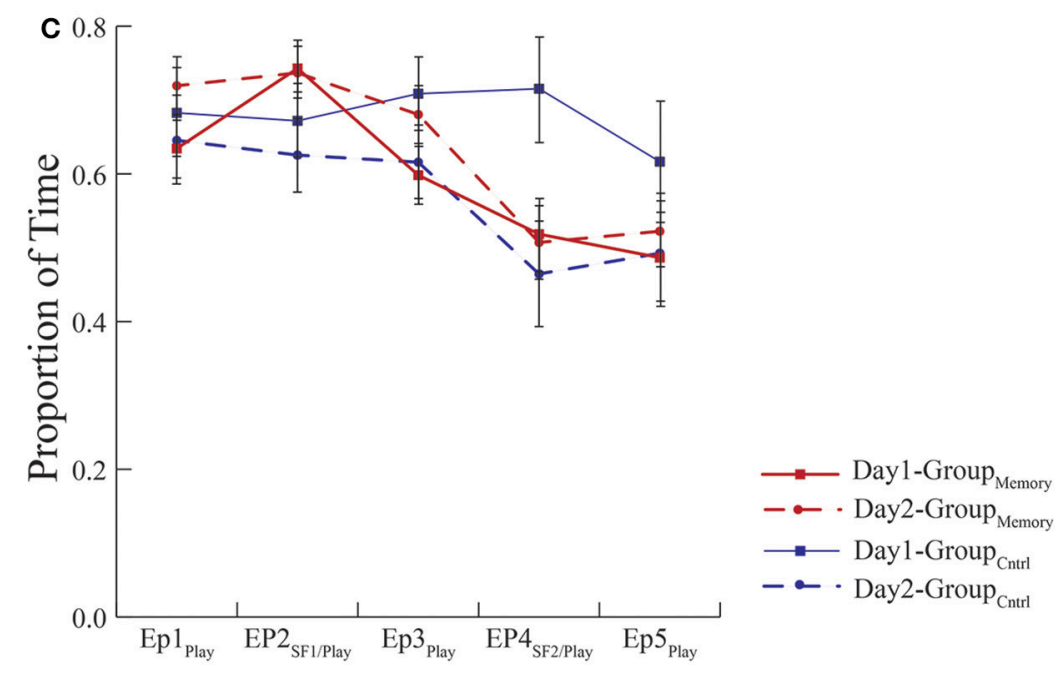

Episode

FIGURE 2 | Mean proportion of time infants spent expressing (A) negative, (B) positive, and (C) neutral affect. All groups exposed to the FFSF paradigm showed the standard SF effect regardless of the number of exposures. There were no differences in behavior indicative of memory between Group Memory infants' first and second exposure to the paradigm or Group Memory infants' second exposure and Groupcontrol infants first exposure to the paradigm (see Day 2 comparisons between groups).

or interactions. Stress response categorization on Day 1 had no effect on Day 2 behavior.

\section{DISCUSSION}

The main goal of this study was to explore potential physiologic and behavioral indices of memory using an empirically validated social stress paradigm, the FFSF paradigm, in a sample of 4month-old infants. Unexpectedly, and unlike previous research, the results of the current study suggest that infants did not remember the social stressor, the SF. There were no changes in cortisol reactivity when comparing Day 2 differences

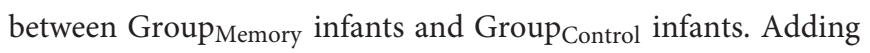
to this, there were no differences in cortisol across days for Group Memory infants. Instead, Group Memory infants were consistent in their peak cortisol reactivity across the 2 days whether showing increases, no change, or decreases in cortisol from baseline.

Similar results were found using cortisol stress reactivity

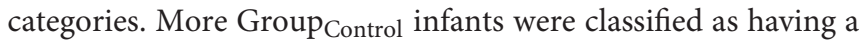
cortisol stress response than Group Memory infants. At face value, this finding might suggest that Group Memory infants remembered the SF experience from Day 1 and were therefore less affected the second time around. However, once again, there was no change in Group Memory infants' cortisol classification over the 2 days. On 
TABLE 6 | Statistical findings for Group Memory infants' behavior across episodes and days.

\begin{tabular}{|c|c|c|c|c|c|c|}
\hline & \multicolumn{3}{|c|}{ Day } & \multicolumn{3}{|c|}{ Episode } \\
\hline & Wilks' $\lambda$ & $\boldsymbol{F}_{(1,27)}$ & $\eta_{\text {partial }}^{2}$ & Wilks' $\lambda$ & $\boldsymbol{F}_{(4,24)}$ & $\eta_{\text {partial }}^{2}$ \\
\hline Gaze to mother & 0.989 & 0.304 & 0.011 & 0.089 & $61.406^{\star \star \star}$ & 0.911 \\
\hline Negative affect & 0.992 & 0.219 & 0.008 & 0.215 & $21.960^{\star \star \star}$ & 0.785 \\
\hline Positive affect & 0.944 & 1.616 & 0.056 & 0.304 & 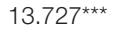 & 0.696 \\
\hline \multirow[t]{3}{*}{ Neutral affect } & 0.942 & 1.674 & 0.058 & 0.360 & $10.648^{\star \star \star}$ & 0.640 \\
\hline & \multicolumn{3}{|c|}{ Episode $\times$ Day } & & & \\
\hline & Wilks' $\lambda$ & $\boldsymbol{F}_{(4,24)}$ & $\eta_{\text {Partial }}^{2}$ & & & \\
\hline Gaze to mother & 0.937 & 0.402 & 0.063 & & & \\
\hline Negative affect & 0.853 & 1.034 & 0.147 & & & \\
\hline Positive affect & 0.708 & $2.469^{\#}$ & 0.292 & & & \\
\hline Neutral affect & 0.835 & 1.184 & 0.165 & & & \\
\hline
\end{tabular}

\# significant at $<0.1$.

*** significant at 0.001

the contrary, the stability of infants' cortisol stress response seems to be a more likely explanation, perhaps due to temperamental differences in reactivity, rather than memory of the social stressor (Kagan and Snidman, 2004).

Stability of infants' responses is also a likely explanation for the behavioral findings. Like cortisol, there were no behavioral changes indicative of memory. When comparing Group Memory

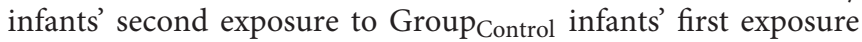
to the FFSF paradigm, Group Control infants were marginally more negative during the first three episodes. However, there were no differences in Group Memory infants' behavior from their first exposure on Day 1 to their second exposure on Day 2. Additionally, when accounting for the relationship between cortisol stress reactivity and behavior across days, there was no difference in Day 2 behavior between infants categorized as having a cortisol stress response on Day 1 and those who were not categorized as having a stress response on Day 1.

The data from this study adds to the growing literature on the double FFSF paradigm as an empirically established social stressor as evidenced by behavioral measures. However, like some previous studies, infants' did not exhibit a cortisol response to their first exposure to the FFSF paradigm (Haley et al., 2011; Montirosso et al., 2013). There was also no relationship between infants' cortisol stress response categorization and behavior during their first exposure to the double FFSF paradigm. In terms of behavior, infants once again demonstrated the standard response to the SF (typical saw-toothed pattern for infant negative affect and the inverse pattern for positive affect and gaze to mother) regardless of the number of exposures to the FFSF paradigm. Infants were also more negative in response to the second SF episode compared to the first thus providing additional support for the double (A-B-A-B-A) FFSF producing more infant distress than the single (A-B-A) FFSF paradigm.

Methodologically speaking, this was one the few FFSF studies to include a control group that was exposed to the laboratory

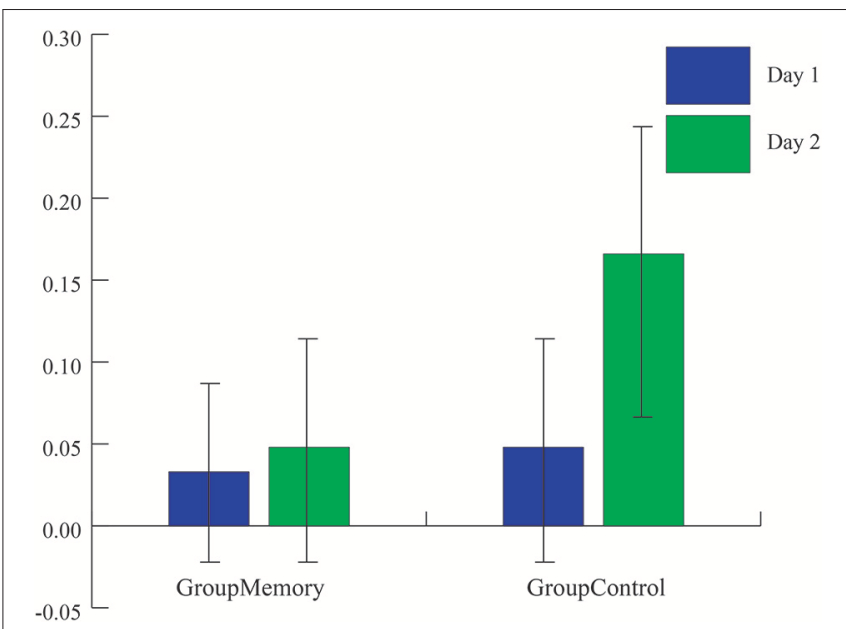

FIGURE 3 | Average peak cortisol reactivity (baseline cortisol value-highest post-paradigm cortisol value) for each group across days. While the average

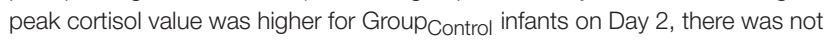
a significant group $x$ day interaction ( $p s>0.1$ ).

but not to the SF on their first visit. Although the control comparison was meant to be a pleasant experience for the dyad, surprisingly Group Control $_{\text {and Group }}$ Memory infants were equally negative on Day 1. However, as seen in Figure 2A, when considering the pattern of negative behavior, Group Memory infants were more negative in response to the SF, especially the second SF, while Group Control infants' negativity increased

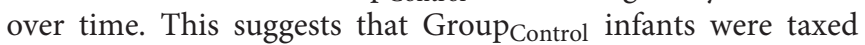
and subsequently dysregulated by the repetitive face-to-face play whereas Group Memory infants were reacting specifically to the SF. The control comparison also makes clear the potential stress brought about by the research experience. It is interesting to note that on Day 1 there was no difference in cortisol between groups (see Figure 3). Both findings suggest that infants were equally stressed on Day 1 as measured by cortisol. One possible interpretation of this finding is that the laboratory experience itself generated a stress response and experiencing the SF did not add to this stress.

Taken together, 4-month-old infants did not demonstrate physiologic or behavioral changes indicative of memory for the FFSF paradigm. Instead, infants' were more likely to demonstrate stability in their response to the social stressor. Why might this be the case? Perhaps the FFSF paradigm was not a strong enough stressor? This does not appear to be the case. Behavioral results suggest the opposite. Group Memory infants demonstrated the classic SF response behaviorally on both days, but did not show an overall increase in cortisol in response to the paradigm. Adding to this, Group Memory infants did not change their stress cortisol categorization across days. Therefore, a more likely explanation of the inconsistency in cortisol findings compared to previous studies points to the emerging or developing response of the adrenocortical system in response to social stress as seen in 4month-old infants. Previous research using this same age group supports this interpretation (Montirosso et al., 2013). 

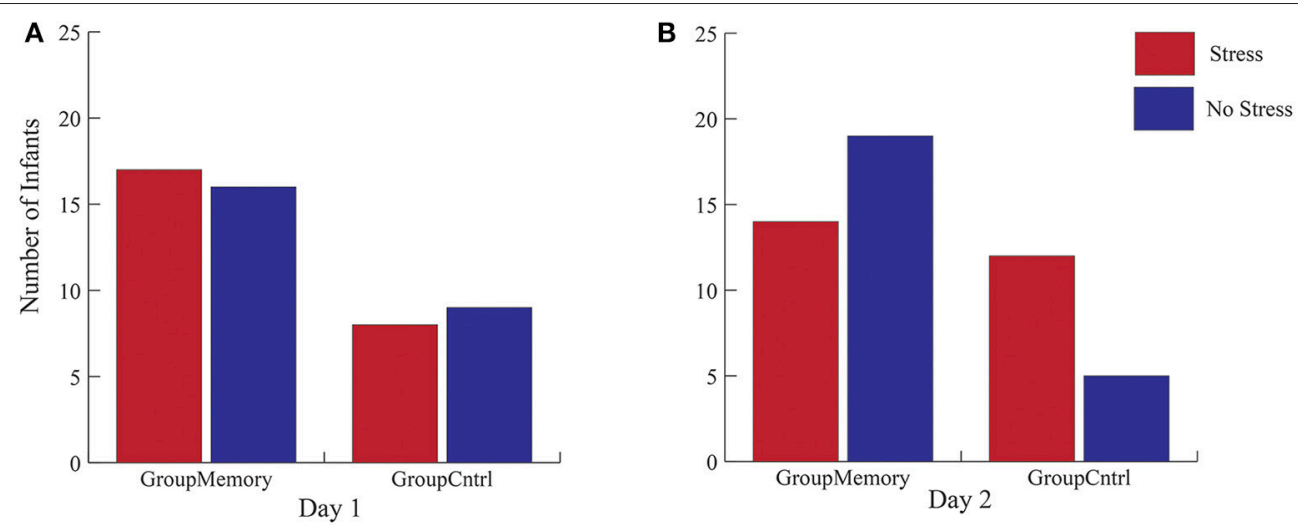

FIGURE 4 | Cortisol stress categorization for both GroupMemory and Group Control infants across days. (A,B) represent the Chi-Square results that compare cortisol categories across groups for each day $(N=50)$.

TABLE 7 | GroupMemory infants' baseline and greater post-paradigm cortisol correlations for Day 1 and Day 2.

\begin{tabular}{|c|c|c|c|c|c|}
\hline & & \multicolumn{2}{|c|}{ Day 1} & \multicolumn{2}{|c|}{ Day 2} \\
\hline & & Baseline & Greater post & Baseline & Greater post \\
\hline \multirow[t]{2}{*}{ Day 1} & Baseline & - & $0.500^{\star}$ & $0.508^{*}$ & $0.467^{\star}$ \\
\hline & Greater post & & - & 0.325 & $0.641^{\star \star}$ \\
\hline \multirow[t]{2}{*}{ Day 2} & Baseline & & & - & $0.553^{\star \star}$ \\
\hline & Greater post & & & & - \\
\hline
\end{tabular}

*significant at the 0.01 level.

**significant at the 0.001 level.

TABLE 8 | GroupMemory statistical findings for main effects of Stress Response Category Day 1 and Episode Day 2 x Stress Response Category Day 1 interactions for all infant measures.

\begin{tabular}{lcccccc}
\hline & $\begin{array}{c}\text { Stress response category } \\
\text { Day 1 }\end{array}$ & & \multicolumn{2}{c}{$\begin{array}{c}\text { Stress response category } \\
\text { Day 1 }\end{array}$} \\
\cline { 2 - 3 } & $\boldsymbol{F}_{(1,26)}$ & $\eta_{\text {partial }}^{2}$ & & Wilks' $\boldsymbol{\lambda}$ & $\boldsymbol{F}_{(4,23)}$ & $\eta_{\text {Partial }}^{2}$ \\
\hline Gaze to mother & 0.351 & 0.013 & & 0.946 & 0.325 & 0.054 \\
Negative affect & 0.005 & $<0.0001$ & & 0.936 & 0.395 & 0.064 \\
Positive affect & 0.736 & 0.028 & & 0.975 & 0.146 & 0.025 \\
Neutral affect & 0.306 & 0.012 & & 0.902 & 0.623 & 0.098 \\
\hline
\end{tabular}

The lack of memory noted in this study stands in stark contrast to the two previous studies. First, there were important methodological and statistical differences when comparing this study to the previous studies. Methodologically speaking, Montirosso et al. (2013) study created cortisol reactivity groups based on any difference between pre- and post-cortisol samples. This way of creating groups did not consider potential assay error. Instead, groups were created by using any difference between pre- and post-cortisol samples. The current study addressed these concerns in the creation of the Stress Response and No Stress Response cortisol groups (see, Granger et al., 2012). The failure to account for error in prior studies could have resulted in an inaccurate representation and interpretation of the cortisol data. Second, there were differences in control comparisons amongst studies. The control comparison group in Montirosso et al. study visited the lab only one time thus ignoring the potential stress brought about by the testing environment itself. The findings from the current study supported the idea of stress generated by the testing environment with almost half of the Group Control infants classified as having a cortisol stress response during the all play episodes on Day 1. Haley et al. (2011) rectified this issue by having a control group visit the lab twice. However, neither the experimental FFSF group nor the control group experienced the FFSF paradigm the second day. Adding to this, there were no cross-day comparisons. Therefore, although Haley et al. found cortisol differences between groups on the second day, the paper lacked within comparisons to isolate variability due to individual differences. Simply put, there was no way of knowing if cortisol responses changed from Day 1 to Day 2. The current paper made both within and between participant and day comparisons to create a more thorough understanding of cortisol reactivity in response to the FFSF paradigm. By repeating visits and exposure to the paradigm, it was possible to tease apart potential effects of the lab and individual differences to better understand differences attributed to memory.

The current study is not without limitations. Though typical of many developmental studies, the sample size was small. The small number of participants most likely contributed to the variability seen in the data, however effect sizes were also small. In addition to finding a large amount of variability in cortisol values, there was also a lot of variability in behavioral responses. Infants became distressed at different points in the paradigm, if at all. Instead of picking an arbitrary point in time to represent the start of the cortisol reactivity clock, perhaps tethering infants' actual distress to the timing of their post-paradigm cortisol samples may reveal a clearer understanding of 4-month-olds' developing neuroendocrine response. The current study only explored one age group using a single paradigm to explore memory for social stress. Therefore, the findings must be interpreted with caution when extending to other age groups and forms of memory. Previous research suggests more consistent cortisol responses 
with older infants (Provenzi et al., 2016) and physical rather than social stress (Gunnar et al., 2009; Jansen et al., 2010) which could be the focus of future studies exploring memory in infancy. Along the same line, when considering memory for socioemotional events, Hertenstein and Campos (2004) found that 14month-olds, but not 11-month-olds, remembered an emotional message following a brief $1 \mathrm{~h}$ delay. DiCorcia and Mumme (reviewed in Mumme et al., 2007) also found that younger infants had a difficult time remembering negative messages. In their study, following an even shorter delay of 15-min, 12-montholds' seemed to remember an actor's focus of attention during a negative reaction but not their emotional intent. Again, the findings from these studies point to a maturation effect in the development of memory for emotional events. Perhaps in the current study with even younger infants, these abilities have yet to come fully online.

In conclusion, while the current study failed to support previous research suggesting that 4-month-olds remembered the SF social stressor, it is important to try to make sense out of the lack of findings given the well-established, highly replicated, stressful behavioral and physiological effects of the FFSF paradigm. On face value, the paradigm should be a good candidate as a memorial event. Yet, here and elsewhere either no memory or only a weak memory effect was found. Contrast that with 4-month-olds' memory for people, events, and actions, what might accounts for the memorial differences? The number and amount of exposures to each might be a possible explanation. In this study, infants were exposed to the FFSF paradigm only once before the memory test. The literature on memory for objects, actions, and people suggests that number and duration of exposure has an effect on memory (Rovee-Collier, 1999; Haley et al., 2011). Certainly the people populating an infant's world are seen often and for longer durations. Perhaps repeated exposures and duration to the FFSF paradigm would have helped spur memory? The lack of findings for what is a well-established stressful event suggests that single exposures to emotional events are not sufficient to produce a long standing memorial effect. Moreover, as established as the stress of the FFSF is, not all infants

\section{REFERENCES}

Adamson, L. B., and Frick, J. E. (2003). The still face: a history of a shared experimental paradigm. Infancy 4, 451-473. doi: 10.1207/S15327078IN0404_01

Carver, L. J., and Bauer, P. J. (2001). The dawning of a past: the emergence of long-term explicit memory in infancy. J. Exp. Psychol. Gen. 130, 726-745. doi: 10.1037/0096-3445.130.4.726

Clements, A. D. (2013). Salivary cortisol measurement in developmental research: where do we go from here? Dev. Psychobiol. 55, 205-220. doi: 10.1002/dev.21025

Cole, P. M., Martin, S. E., and Dennis, T. A. (2004). Emotion regulation as a scientific construct: methodological challenges and directions for child development research. Child Dev. 75, 317-333. doi: 10.1111/j.1467-8624.2004.00673.x

Giles, A., and Rovee-Collier, C. (2011). Infant long-term memory for associations formed during mere exposure. Infant Behav. Dev. 34, 327-338. doi: 10.1016/j.infbeh.2011.02.004

Granger, D. A., Fortunato, C. K., Beltzer, E. K., Virag, M., Bright, M. A., and Out, D. (2012). Focus on methodology: salivary bioscience and react to it. There are striking individual differences. Adding to this, differences in cortisol reactivity have also been noted across early infancy (Jansen et al., 2010; Clements, 2013; Hill-Soderlund et al., 2014; Martinez-Torteya et al., 2015). Thus, one must be cautious in their attributions of permanent long- term effects of a singular stressful event because of both unknown developmental parameters that might affect the creation of long-term memories and individual differences in reactivity to the same event. Future studies should focus on these issues to gain a fuller understanding of the development of both the adrenocortical response and memory in infancy.

\section{ETHICS STATEMENT}

This study was carried out in accordance with the recommendations of the Institutional Review Board at the University of Massachusetts, Boston. All participants (mothers) gave written informed consent in accordance with the Declaration of Helsinki. The protocol was approved by the University of Massachusett's Institutional Review Board.

\section{AUTHOR CONTRIBUTIONS}

All authors were involved in the design of the project and oversaw data collection. The corresponding author conceptualized and carried out data analyses and drafted the paper. All authors worked together toward the final manuscript.

\section{ACKNOWLEDGMENTS}

This research was funded by grants from the Eunice Kennedy Shriver NICHD (R01 HD050459-01) and the National Science Foundation (06-511). We would like to thank our research team at the Child Development Unit, especially Lauren Balest, Emily Brown, Erin Duffy, Dominick Newell, and Emily Ogoreuc, for their contributions to data collection and coding. Thank you to all of our families for their time, participation, and interest. research on adolescence: an integrated perspective. J. Adolesc. 35, 1081-1095. doi: 10.1016/j.adolescence.2012.01.005

Granger, D. A., Hibel, L. C., Fortunato, C. K., and Kapelewski, C. H. (2009). Medication effects on salivary cortisol: tactics and strategy to minimize impact in behavioral and developmental science. Psychoneuroendocrinology 34, 1437-1448. doi: 10.1016/j.psyneuen.2009.06.017

Gunnar, M. R., Talge, N. M., and Herrera, A. (2009). Stressor paradigms in developmental studies: what does and does not work to produce mean increases in salivary cortisol. Psychoneuroendocrinology 34, 953-967. doi: 10.1016/j.psyneuen.2009.02.010

Haley, D. W., Cordick, J., Mackrell, S., Antony, I., and Ryan-Harrison, M. (2011). Infant anticipatory stress. Biol. Lett. 7, 136-138. doi: 10.1098/rsbl.2010.0565

Haley, D. W., and Stansbury, K. (2003). Infant stress and parent responsiveness: regulation of physiology and behavior during still-face and reunion. Child Dev. 74, 1534-1546. doi: 10.1111/1467-8624.00621

Hartshorn, K., Rovee-Collier, C., Gerhardstein, P., Bhatt, R. S., Wondoloski, T. L., Klein, P., et al. (1998). The ontogeny of long-term memory over the first year-and-a-half of life. Dev. Psychobiol. 32, 69-89. doi: 10.1002/(SICI)1098-2302(199803)32:2<69::AID-DEV1>3.0.CO;2-Q 
Hertenstein, M. J., and Campos, J. J. (2004). The retention effects of an adult's emotional displays on infant behavior. Child Dev. 75, 595-613. doi: 10.1111/j.1467-8624.2004.00695.x

Hibel, L. C., Granger, D. A., Kivlighan, K. T., and Blair, C. (2006). Individual differences in salivary cortisol: associations with common over-the-counter and prescription medication status in infants and their mothers. Horm. Behav. 50, 293-300.doi: 10.1016/j.yhbeh.2006.03.014

Hill-Soderlund, A. L., Holochwost, S. J., Willoughby, M. T., Granger, D. A., Gariépy, J.-L., Mills-Koonce, W. R., et al. (2014). Developmental course of salivary alpha-amylase and cortisol from 12 to 36 months: relations with early poverty and later behavior problems. Psychoneuroendocrinology 52, 1-13. doi: 10.1016/j.psyneuen.2014.08.011

Howell, D. C. (2002). Statistical Methods for Psychology. Pacific Grove, CA: Wadsworth Group.

Jansen, J., Beijers, R., Riksen-Walraven, M., and de Weerth, C. (2010). Cortisol reactivity in young infants. Psychoneuroendocrinology 35, 329-338. doi: 10.1016/j.psyneuen.2009.07.008

Kagan, J., and Snidman, N. C. (2004). The Long Shadow of Temperament. Cambridge, MA: Belknap Press; Harvard University Press.

Learmonth, A. E., Lamberth, R., and Rovee-Collier, C. (2004). Generalization of deferred imitation during the first year of life. J. Exp. Child Psychol. 88, 297-318. doi: 10.1016/j.jecp.2004.04.004

Lewis, M., and Ramsay, D. (2005). Infant emotional and cortisol responses to goal blockage. Child Dev. 76, 518-530. doi: 10.1111/j.1467-8624.2005.0 0860.x

Lukowski, A. F., and Bauer, P. J. (eds.). (2013). The Wiley Handbook on the Development of Children's Memory. Chichester: John Wiley \& Sons Ltd.

Martinez-Torteya, C., Muzik, M., McGinnis, E. W., Rosenblum, K. L., Bocknek, E. L., Beeghly, M., et al. (2015). Longitudinal examination of infant baseline and reactivity cortisol from ages 7 to 16 months. Dev. Psychobiol. 57, 356-364. doi: 10.1002/dev.21296

Montirosso, R., Tronick, E., Morandi, F., Ciceri, F., and Borgatti, R. (2013). Fourmonth-old infants' long-term memory for a stressful social event. PLoS ONE 8:e82277. doi: 10.1371/journal.pone.0082277

Mumme, D. L., Bushnell, E. W., DiCorcia, J. A., and Adams Lariviere, L. (2007). "Infants' use of gaze cues to interpret others' actions and emotional reactions," in Gaze-Following: Its Development and Significance, eds R. A. Flom, K. Lee, and D. W. Muir (Mahwah, NJ: Lawrence Erlbaum Associates, Inc.), 143-170.

Pascalis, O., and de Schonen, S. (1994). Recognition memory in 3- to 4-day-old human neonates. Neuroreport 5, 1721-1724. doi: 10.1097/00001756-199409080-00008
Provenzi, L., Giusti, L., and Montirosso, R. (2016). Do infants exhibit significant cortisol reactivity to the face-to-face still-face paradigm? a narrative review and meta-analysis. Dev. Rev. 42, 34-55. doi: 10.1016/j.dr.2016.07.001

Ramsay, D., and Lewis, M. (2003). Reactivity and regulation in cortisol and behavioral responses to stress. Child Dev. 74, 456-464. doi: 10.1111/1467-8624.7402009

Rovee-Collier, C. (1999). The development of infant memory. Curr. Dir. Psychol. Sci. 8, 80-85. doi: 10.1111/1467-8721.00019

Stack, D. M., and Muir, D. W. (1990). Tactile stimulation as a component of social interchange: new interpretations for the still-face effect. Br. J. Dev. Psychol. 8 , 131-145. doi: 10.1111/j.2044-835X.1990.tb00828.x

Toda, S., and Fogel, A. (1993). Infant response to the still-face situation at 3 and 6 months. Dev. Psychol. 29, 532-538. doi: 10.1037/0012-1649.29.3.532

Tollenaar, M. S., Jansen, J., Beijers, R., Riksen-Walraven, J. M., and de Weerth, C. (2010). Cortisol in the first year of life: normative values and intra-individual variability. Early Hum. Dev. 86, 13-16. doi: 10.1016/j.earlhumdev.2009.12.003

Tronick, E., Als, H., Adamson, L., Wise, S., and Brazelton, T. B. (1978). The infant's response to entrapment between contradictory messages in face-to-face interaction. J. Am. Acad. Child Psychiatry 17, 1-13. doi: 10.1016/S0002-7138(09)62273-1

Weinberg, M. K., and Tronick, E. Z. (1996). Infant affective reactions to the resumption of maternal interaction after the still-face. Child Dev. 67, 905-914. doi: $10.2307 / 1131869$

Weinberg, M. K., and Tronick, E. Z. (1999). Infant and Caregiver Engagement Phases (ICEP) Scoring System. Boston, MA: Child Development Unit Children's Hospital Boston and Harvard Medical School.

Weinberg, M. K., Tronick, E. Z., Cohn, J. F., and Olson, K. L. (1999). Gender differences in emotional expressivity and self-regulation during early infancy. Dev. Psychol. 35, 175-188. doi: 10.1037/0012-1649.35.1.175

Conflict of Interest Statement: The authors declare that the research was conducted in the absence of any commercial or financial relationships that could be construed as a potential conflict of interest.

Copyright (c) 2018 DiCorcia, Snidman and Tronick. This is an open-access article distributed under the terms of the Creative Commons Attribution License (CC $B Y)$. The use, distribution or reproduction in other forums is permitted, provided the original author(s) and the copyright owner are credited and that the original publication in this journal is cited, in accordance with accepted academic practice. No use, distribution or reproduction is permitted which does not comply with these terms. 